\title{
Thermal properties in strong hydrogen bonding systems composed of poly(vinyl alcohol), polyethyleneimine, and graphene oxide
}

\author{
Sua Choi ${ }^{1}$, Duck Kun Hwang ${ }^{2}$ and Heon Sang Lee ${ }^{1, \mathrm{~A}}$ \\ ${ }^{1}$ Department of Chemical Engineering, Dong-A University, Busan 604-714, Korea \\ ${ }^{2}$ Department of Corporate Diagnosis, Small \& Medium Business Corporation, Seoul 150-718, Korea
}

\author{
Article Info \\ Received 11 July 2014 \\ Accepted 25 September 2014 \\ *Corresponding Author \\ E-mail: heonlee@dau.ac.kr \\ Tel: $+82-51-200-7724$

\section{Open Access} \\ DOI: http://dx.doi.org/ \\ 10.5714/CL.2014.15.4.282 \\ This is an Open Access article distributed \\ under the terms of the Creative Commons \\ Attribution Non-Commercial License \\ (http://creativecommons.org/licenses/ \\ by-nc/3.0/) which permits unrestricted \\ non-commercial use, distribution, and \\ reproduction in any medium, provided \\ the original work is properly cited.
}

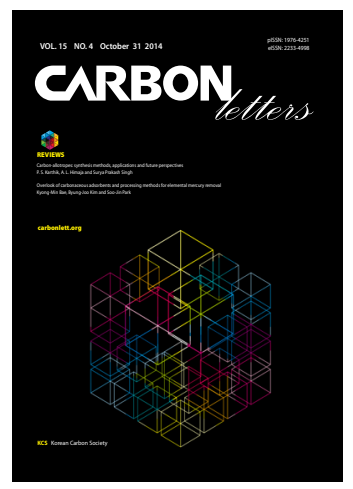

http://carbonlett.org

pISSN: $1976-4251$

elSSN: 2233-4998

Copyright $\odot$ Korean Carbon Society

\begin{abstract}
Blends of poly(vinyl alcohol) (PVA), polyethyleneimine (PEI), and graphene oxide (GO) were prepared by solution casting method. Calorimetric thermal properties of the blends were investigated. The $T_{g} \mathrm{~s}$ of PVA/PEI blends were higher than the $T_{g} \mathrm{~s}$ of either of the component polymers at low concentrations of PEI. These abnormal increases of $T_{g} \mathrm{~s}$ may be due to the negative entropy of mixing which is associated with strong hydrogen bonding between PVA and PEI. The degree of depression of $T_{m}^{0}$ s was not reduced by the negative entropy of mixing, since strong hydrogen bonding also causes an increase in the magnitude of negative $\chi$ between PVA and PEI. The $T_{g}$ of PVA was increased significantly by adding $0.7 \mathrm{wt} . \%$ GO into PVA. The magnitude of negative $\chi$ was increased by adding GO into the blends of PVA and PEI.
\end{abstract}

Key words: poly(vinyl alcohol), polyethyleneimine, graphene oxide, calorimetric thermal properties, strong hydrogen bonding

\section{Introduction}

Poly(vinyl alcohol) (PVA) and its blend with polyethyleneimine (PEI) have attracted great interest due to their potential application in functional membranes as well as for fibers [1-8]. The blends are also a good model system for miscible polymer blends with strong hydrogen bonding. Graphene oxide (GO) is a chemically exfoliated graphite, which is a precursor for graphene (or reduced GO) sheets [9-11]. Graphene has emerged as an ingredient for polymer nanocomposites due to its exceptional electron transport [12-14], mechanical and rheological properties [15-17], and gas barrier properties [15,18,19]. Molecular level dispersion of GO has been reported in the PVA/GO system, which may be ascribed to the hydrogen bonding interaction between the components [18,20].

Hydrogen bonding in polymer blends has been of interest to polymer scientists for several decades [21-39]. One major characteristic in such systems is the large positive deviation of glass transition temperature $\left(T_{g}\right)$ from conventional mixing rules [22,23,26,35]. It is well known that miscible binary blends exhibit a single $T_{g}$ between the $T_{g} S$ of their individual component polymers. Many expressions have been proposed for evaluating the $T_{g} s$ of miscible binary blends, such as Fox [40], Gordon and Taylor [41], Couchman and Karasz [42], and Kwei [43] equation. Most of these expressions predict a single $T_{g}$ located in between the $T_{g} s$ of the component polymers. However, the $T_{g} s$ of blends of polyvinylphenol (PVPh) and polyvinylpyridine (PVPy) are higher than those of both components due to the strong hydrogen bonding [37]. Two different views on the abnormal increase of $T_{g}$ have been reported. Painter and coworkers [37] claimed that the abnormal increase of $T_{g}$ can be ascribed to the positive enthalpy of mixing (endothermic) by strong hydrogen bonding, while the hydrogen 
bonding itself is exothermic. Pinal [22] have extended the classical thermodynamic approach of Couchman and Karasz [42] to the hydrogen bonding system, and reached the conclusion that the abnormal increase of $T_{g}$ is due to the negative entropy of mixing. Either case, the negative entropy of mixing or endothermic enthalpy of mixing is certainly abnormal behavior induced by strong hydrogen bonding.

At equilibrium melting temperature, the chemical potential of the crystalline phase is equal to that of the liquid phase $[44,45]$. If the crystalline phase sustains the pure state, a new equilibrium condition arises where the chemical potential of the crystalline phase is equal to the reduced-chemical potential of the mixedliquid phase, which leads to the depression of equilibrium melting temperature [45]. Therefore, either the negative entropy of mixing or the positive enthalpy of mixing by strong hydrogen bonding may weaken the depression of melting temperature. Unfortunately, the melting point depressions could not be examined in the amorphous blends of PVPh and PVPy by Painter and coworkers [37]. Subsequently, Painter and coworkers [46] reported the effect of hydrogen bonding on the melting point depression in polymer blends where one component crystallizes. However, the abnormal increase of $T_{g}$ was not investigated in the latter case.

Blends of PVA/PEI may be a good alternative model system for investigating both melting behaviors and glass transition temperatures, since PVA is a semi-crystalline polymer. PEI is a multifunctional aliphatic amine with strong cationic characteristics, whereas PVA is a water-soluble poly hydroxyl polymer. Amino groups in the PEI chain could also form strong hydrogen bonds with hydroxyl groups in the PVA chain.

In this work, we have explored the thermal properties of PVA/ $\mathrm{PEI} / \mathrm{GO}$ blend systems. In particular, we investigated the glass transition temperatures and the equilibrium melting temperatures. We evaluated the configurational entropy of mixing from the glass transition temperatures in the strong hydrogen bonding systems. The effect of strong hydrogen bonding on the degree of depression of equilibrium melting points was also investigated. We also examined the effect of GO on the thermal behaviors of PVA/PEI blends.

\section{Experimental Section}

\subsection{Materials}

PVA was purchased from Sigma Aldrich (99 mol\% hydrolyzed, $\left.\mathrm{M}_{\mathrm{w}}=89000-98000 \mathrm{~g}\right)$. PEI, $50 \mathrm{wt} \%$ aqueous solution with the low molecular weight of 2000 including amino groups (ratio of primary/secondary/tertiary amino groups was roughly 1/2/1), was purchased from Sigma Aldrich. Flake graphite powder (19 $\mu \mathrm{m}$ nominal particle size) was supplied from Asbury Carbon. GO was synthesized from purified conventional flake graphite by modified Hummers method [9] as reported in our earlier work $[17,18]$.

\subsection{PVA/PEI/GO blend preparation}

PVA solution was prepared by dissolving pure PVA powder with distilled water at $363 \mathrm{~K}$ for $1 \mathrm{~h}$ and stirring at the same time. Then, the PVA solution was cooled to room temperature and mixed with PEI aqueous solution at a designated mixing ratio by stirring for $1 \mathrm{~h}$. A homogenized solution was prepared in a bubble-free state. A colloidal solution of GO was added to the $\mathrm{PVA} / \mathrm{PEI}$ solution with a designated mixing ratio and the PVA/ $\mathrm{PEI} / \mathrm{GO}$ solution was milled for $0.5 \mathrm{~h}$ at $298 \mathrm{~K}$. Then, the solution was cast on glass plate at $373 \mathrm{~K}$ and dried in a convection oven at $373 \mathrm{~K}$ for $3 \mathrm{~h}$ followed by drying in a vacuum oven at $393 \mathrm{~K}$ for $3 \mathrm{~h}$ to ensure the removal of moisture.

\subsection{Characterization of PVA/PEI blends}

The morphology of the blends was characterized with field emission scanning electron microscopy (FE-SEM; JEOL JSM$6700 \mathrm{~F}$ ) by observation of the cryogenically fractured surface of samples. High-resolution transmission electron microscopy (HRTEM) was performed with a Hitachi HF-2000 operated at $200 \mathrm{kV}$. Samples were applied onto a 400 mesh $\mathrm{Cu}$ grid with lacey carbon film and dried in air before TEM imaging. X-ray photoelectron spectroscopy (XPS) was performed in a Multilab2000 with a Mg-K X-ray source using a power source of $300 \mathrm{~W}$. The atomic fractions of the different elements in the $10 \mathrm{~nm}$ upper layer were probed by XPS and calculated from the survey spectra. X-ray diffraction (XRD) analyses were performed directly on the hybrid samples using a Rigaku (Japan)Ultima IV (XRD; $40 \mathrm{kV}, 40 \mathrm{~mA}$ ) with $\mathrm{Cu}$ irradiation at a scanning rate of $0.02 / \mathrm{s}$ in the $2 \theta$ range of $2-40$. The non-isothermal melting temperatures and glass transition temperatures were measured by differential scanning calorimetry (TA-DSC) from 203 to $523 \mathrm{~K}$ during a 2 nd run with a heating rate of $20 \mathrm{~K} / \mathrm{min}$. To investigate isothermal crystallization and melting, samples were heated to $523 \mathrm{~K}$, held there for $5 \mathrm{~min}$, and cooled rapidly $(-80 \mathrm{~K} / \mathrm{min})$ to the designated crystallization temperature. When the isothermal crystallization procedure was finished (as monitored by exothermic peaks during isothermal crystallization), the samples were heated again to $523 \mathrm{~K}$ with a heating rate of $20 \mathrm{~K} / \mathrm{min}$.

\section{Results and Discussion}

\subsection{Glass transition temperatures $\left(T_{g} S\right)$ of PVA/ PEI blends}

DSC thermograms for PVA/PEI blends are seen in Fig. 1. The glass transition temperatures $\left(T_{g} S\right)$, onset points $\left(T_{1} S\right)$ and end points $\left(T_{2} s\right)$ of glass transitions are listed in were obtained by DSC thermograms. The composition dependency of $T_{g} s$ of the PVA/ PEI binary blend is presented in Fig. 2a. The calculation results by Couchman-Karasz equation are compared to the experimental data in Fig. 2a. The Couchman and Karasz equation [42] is

$$
\ln T_{C K}=\frac{x_{1} \Delta C_{p 1} \ln T_{g 1}+x_{2} \Delta C_{p 2} \ln T_{g 2}}{x_{1} \Delta C_{p 1}+x_{2} \Delta C_{p 2}}
$$

where $T_{C K}, T_{g 1}$, and $T_{g 2}$ is the glass transition temperature of the mixture, PEI, and PVA, respectively. $x_{1}$ and $x_{2}$ are the mole fractions of PEI and PVA, respectively. $\Delta C_{p i}$ is the difference in the heat capacity of the liquid and the heat capacity of the glass forms of component $i$. Molar volumes of the repeating unit of PEI $\left(\mathrm{V}_{1 \mathrm{u}}=36.11 \mathrm{~cm}^{3} / \mathrm{mol}\right)$ and PVA $\left(\mathrm{V}_{2 \mathrm{u}}=32.28 \mathrm{~cm}^{3} / \mathrm{mol}\right)$ were 


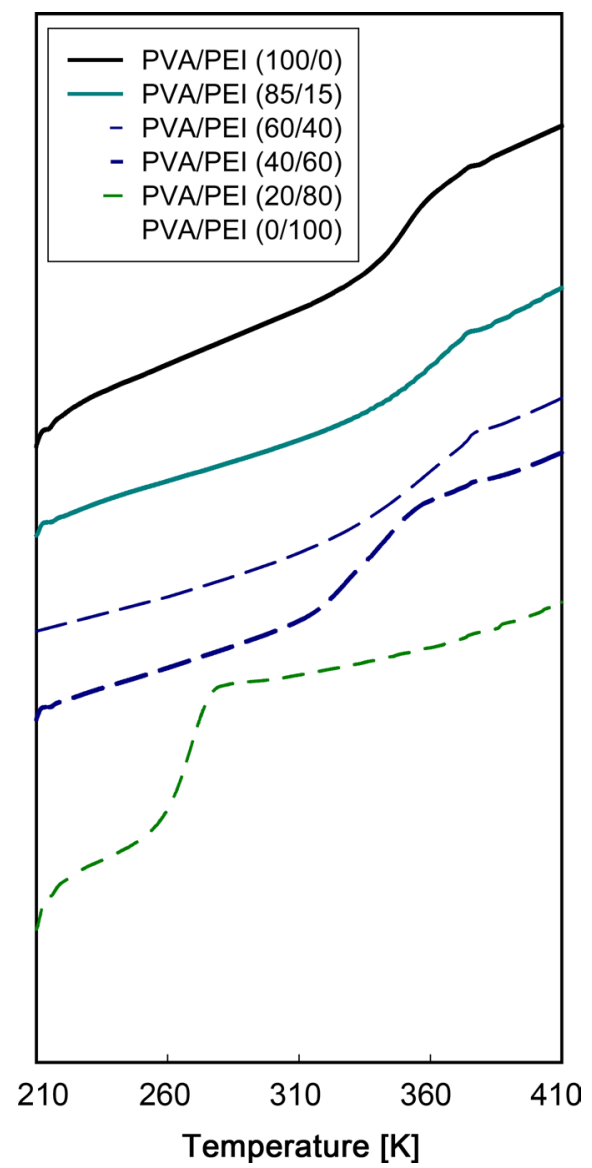

Fig. 1. Differential scanning calorimetry thermograms for the glass transition temperatures of the blends of poly(vinyl alcohol) (PVA) and polyethyleneimine (PEI).

used to estimate mole fractions $x_{\mathrm{i}}$.

A large positive deviation from the calculated values can be observed in Fig. 2a. Specifically, the $T_{g} s$ of the PVA/PEI blends are higher than either of the component $T_{g} s$ at low concentrations of PEI in Fig. 2a. These abnormal increases of $T_{g} s$ may be due to the strong hydrogen bonding between PVA and PEI, which is a rarely observed behavior in polymer blend systems.

According to Gibbs and DiMarzio [47], the glass transition temperature may be defined as the point where the configurational entropy becomes zero when a polymer in melt state is cooled down to a glassy state. OH--N bonding may be formed in PVA/PEI blends, as seen in Fig. $2 b$, which may be stronger than OH--O bonding in PVA itself or than NH--N bonding in PEI. So, the strengthened hydrogen bonding produced by mixing may induce a decrease of the configurational entropy in the blend at melt state. This may lead to the abnormal increase of $T_{g} s$. Pinal [22] has extended the classical thermodynamic approach of Couchman-Karasz to a hydrogen bonding system and has obtained following expression.

$$
T_{g m}=T_{C K} \exp \left(-\frac{\Delta S_{m i x}^{c}}{x_{1} \Delta C_{p 1}+x_{2} \Delta C_{p 2}}\right)
$$

where $T_{g m}$ is the glass transition temperature of the mixture obtained experimentally, $T_{C K}$ is defined in Eq. 1 , and $\Delta S_{m i x}^{c}$ is the

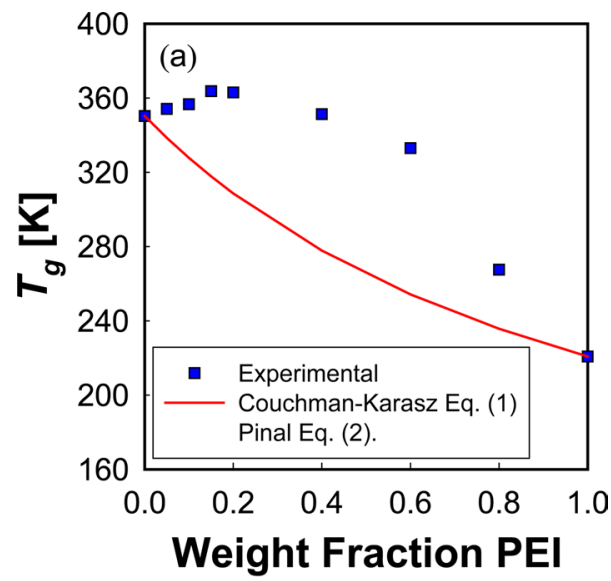

(b)

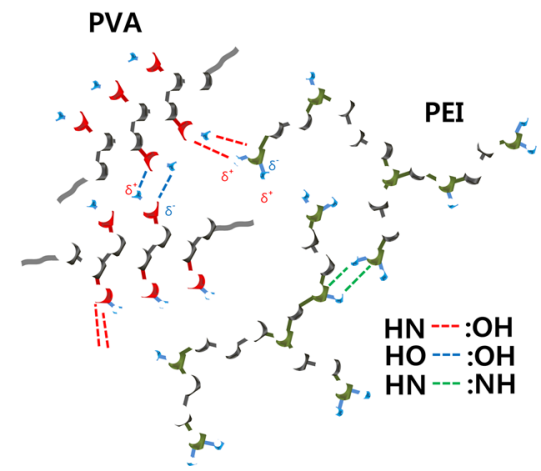

Fig. 2. Composition dependency of the glass transition glass transition temperatures of the blends of poly(vinyl alcohol) (PVA) and polyethyleneimine (PEI); (a) the $T_{g} S$ with respect to the weight fraction of PEI, (b) schematic illustration for the hydrogen bondings in the blends of PVA and PEI.

configurational part of the entropy of mixing. We determined $\Delta S_{m i x}^{c}$ by applying Eq. (2) to the experimental data in Fig. 2a. The estimated $\Delta S_{\text {mix }}^{c}$ has a negative sign as seen in Fig. 3a. The abnormal increases of $T_{g} s$ in Fig. 2a are ascribed to the negative configurational entropy of mixing, which may be caused by the formation of strong hydrogen bonding between PVA and PEI.

The entropy change of the PVA/PEI blends in the glass transition region can be obtained by Fig. $3 \mathrm{~b}$ according to the definition of entropy by Clausius [44].

$$
\Delta S_{g}=\int_{T_{1}}^{T_{2}} \frac{\Delta C_{p}}{T} d T=\Delta C_{p} \ln \left(\frac{T_{2}}{T_{1}}\right)
$$

$\Delta S_{g}$ was evaluated from the experimental data in Table 1 and Fig. 2a. The $\Delta S_{g}$ shows an S-shaped curve, as seen in Fig. 3b. The $\Delta S_{g}$ is initially decreased by adding PEI into PVA, up to a 0.2 weight fraction. $\Delta S_{g}$ reaches maximum value around the mid-composition range, then is decreased by the further increase of PEI weight fraction, in Fig. 4. The abnormal increase of $T_{g} s$ in Fig. 2a may also be consistent with the decrease of $\Delta S_{g}$ at low concentration range in Fig. 3b, where the weight fraction of PEI is less than 0.2 . 

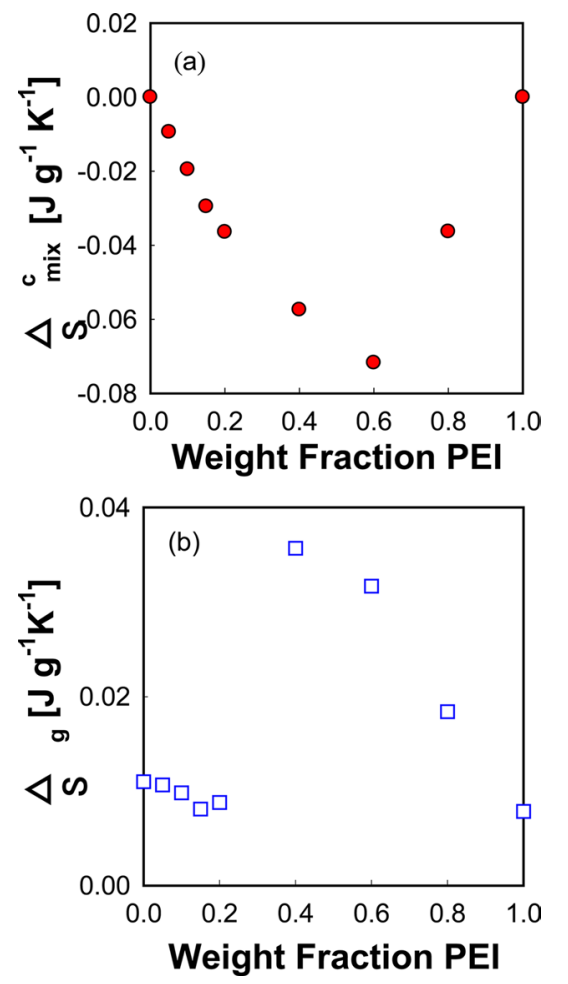

Fig. 3. Entropy of mixing of the blends of poly(vinyl alcohol) and polyethyleneimine (PEI); (a) configurational entropy of mixing, (b) entropy in the glass transition region.

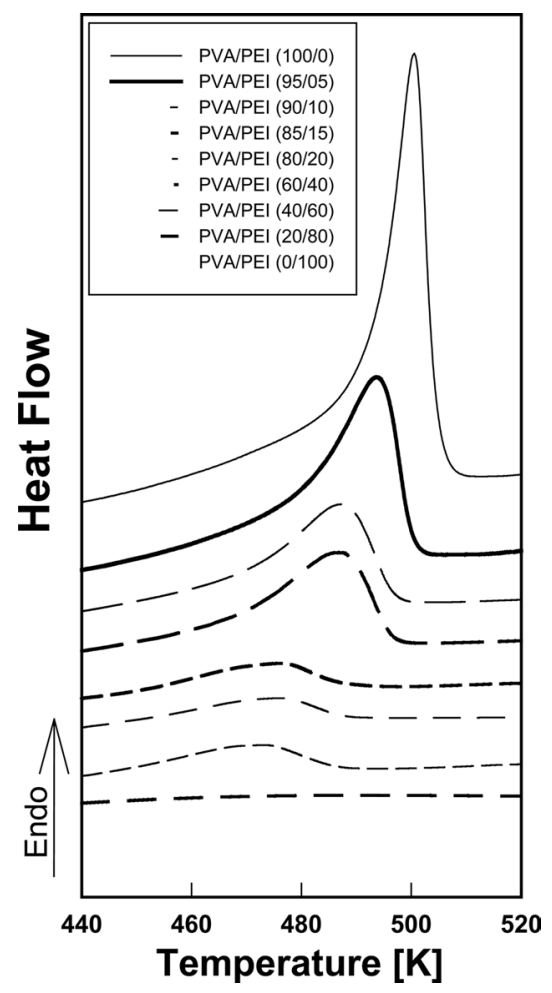

Fig. 4. Differential scanning calorimetry thermograms for the melting temperatures of poly(vinyl alcohol) (PVA) in the blends of PVA and polyethyleneimine (PEI).
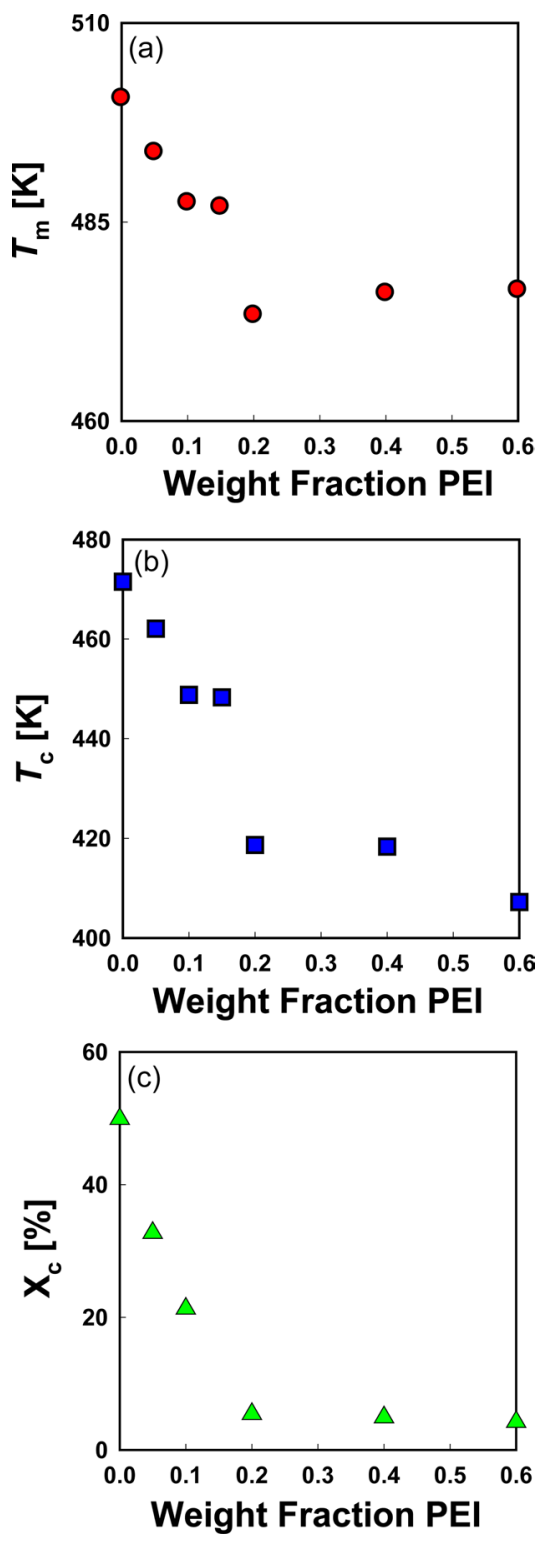

Fig. 5. Non-isothermal properties of poly(vinyl alcohol) (PVA) in the blends of PVA and polyethyleneimine (PEI) with the heating or cooling rate of $20 \mathrm{~K} / \mathrm{min}$ in differential scanning calorimetry; (a) melting temperatures, (b) crystallization temperatures, (c) crystallinities.

\subsection{Melting behaviors of PVA/PEI blends}

DSC thermograms for melting peaks of PVA in PVA/PEI blends are presented in Fig. 4. The non-isothermal melting temperatures $\left(T_{m} s\right)$ were measured from the 2 nd heating with a heating rate of $20 \mathrm{~K} / \mathrm{min}$. The crystalline temperatures $\left(T_{c} s\right)$ were measured with the cooling rate of $20 \mathrm{~K} / \mathrm{min}$. Crystallinity $\left(X_{c}\right)$ of the blends was evaluated with the equation below.

$$
X_{c}=\frac{\Delta H_{f}}{\Delta H_{f}^{o}}
$$

where $X_{c}$ is the crystallinity, $\Delta H_{f}$ is the heat of fusion of the blends and $\Delta H_{f}^{o}$ is the heat of fusion of $100 \%$ crystalline polymer, PVA 

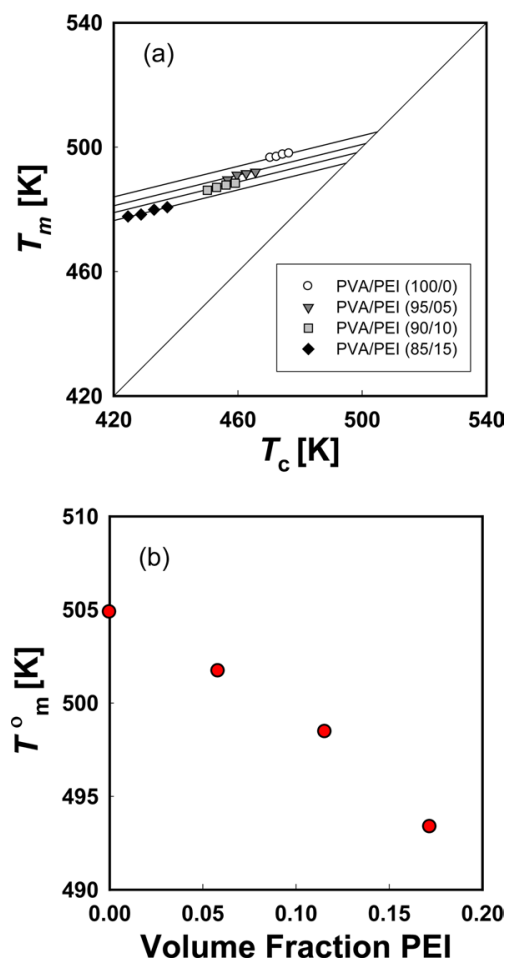

Fig. 6. Equilibrium melting temperatures of poly(vinyl alcohol) (PVA) in the blends of PVA and polyethyleneimine (PEI); (a) Hoffman-Weeks plot, (b) composition dependency of equilibrium melting temperatures of PVA.

(138.6 J/g) [31]. In Fig. 5a-c, the $T_{m} s, T_{c} s$, and $X_{c} s$ are remarkably decreased by the addition of PEI up to 0.2 weight fraction, and are changed slightly by the further addition of PEI into PVA. When the weight fraction of PEI was higher than 0.8 , melting peaks were not observed by DSC in the PVA/PEI blends. The composition range associated with the large decreases of $T_{m} s$, $T_{c} s$, and $X_{c} s$ in Fig. 5 is consistent with the composition range associated with the abnormal increases of $T_{g} s$ in Fig. 2a.

Fig. 6a is a Hoffman-Week's plot [48] for the estimation of equilibrium melting temperature $\left(T_{m}\right)$ of PVA in blends of PVA and PEI. The composition dependency of $T_{m}$ is plotted in Fig. 6b. At equilibrium melting temperature, the chemical potential of crystalline PVA $\left(\mu_{c}\right)$ is equal to those of PVA melt $\left(\mu_{l}\right)$. The $\mu_{l}$ may be reduced to $\mu_{l}$ by adding PEI into PVA, since PVA may be mixed with PEI spontaneously. Then, one can obtain the following equilibrium condition [45].

$$
\mu_{c}-\mu_{l}=\mu_{l}^{*}-\mu_{l}
$$

The left hand side of Eq. (5) is the molar free energy of fusion $\left(\Delta F_{f}=-\Delta H_{f}\left(1-T_{m} / T_{m}^{0}\right)\right)$ and the right hand side is the partial

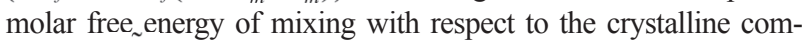
ponent $\left(\Delta F_{m}\right)$. In the case of a hydrogen-bonding system, the free energy of mixing can be divided into two terms, as in Eq. (6) [37].

$$
\Delta \tilde{F}_{m}=\Delta \tilde{F}_{m}^{P}+\Delta \tilde{F}_{m}^{H}
$$

where ${\tilde{\Delta F_{m}}}_{m}$ and $\tilde{\Delta}_{m}^{P}$ is the partial molar free energy of mixing contributed by the hydrogen bonding force and by all other forces, respectively. Usually, $\Delta \tilde{F}_{m}^{P}$ is given by the Flory-Huggins equation. Eq. (6) can be re-written as Eq. (7).

$$
\Delta \tilde{F}_{m}=\frac{\partial F_{m i x}}{\partial n_{2}}=\left(\tilde{H}_{m}^{P}+\Delta \tilde{H}_{m}^{H}\right)-T \Delta \tilde{S}_{m}
$$

where $n_{2}$ is the number of moles of the repeating unit of PVA. According to Nish and Wang [49], the enthalpy of mixing can be given by Eqs. (8) and (9) by neglecting the effect of $\phi_{2}$ in the Flory-Huggins equation.

$$
\begin{aligned}
& \Delta \tilde{H}_{m}=\frac{\partial H_{m i x}}{\partial n_{2}}=\left(\frac{V_{2 u}}{V_{1 u}}\right) R T \phi_{1}^{2}\left(\chi^{P}+\chi^{H}\right)=\left(\frac{V_{2 u}}{V_{1 u}}\right) R T \phi_{1}^{2} \chi \\
& \chi=\frac{B V_{1 u}}{R T}
\end{aligned}
$$

where $B$ is the interaction energy density characteristic of the polymer pair. The following expression can be obtained by inserting Eq. (8) into Eq. (7).

$$
\frac{\Delta \tilde{F}_{m}}{R T}=-\frac{\Delta \tilde{S}_{m}}{R}+\phi_{1}^{2}\left(\frac{V_{2 u}}{V_{1 u}}\right)\left(\chi^{P}+\chi^{H}\right)=-\frac{\Delta \tilde{S}_{m}}{R}+\phi_{1}^{2}\left(\frac{V_{2 u}}{V_{1 u}}\right) \chi
$$

By setting $\Delta \tilde{F}_{m}$ equal to the molar free energy of fusion $\left(\Delta \tilde{F}_{f}=-\Delta H_{f}\left(1-T_{m} / T_{m}^{0}\right)\right)$ and inserting Eq. (9) into Eq. (10), the following expressions can be obtained

$$
\frac{1}{\phi_{1}}\left(\frac{1}{T_{m}}-\frac{1}{T_{m}^{0}}\right)-\left(\frac{\Delta \tilde{S}_{m}}{\phi_{1}} \frac{1}{\Delta \tilde{H}_{f}}\right)=-\frac{\phi_{1}}{T_{m}} \frac{B V_{2 u}}{\Delta \tilde{H}_{f}}
$$

where $\Delta \tilde{S}_{m}$ is athermal in the liquid state. We refer to the left hand side of Eq. (11) as $Q$ as follows.

$$
Q=\frac{1}{\phi_{1}}\left(\frac{1}{T_{m}}-\frac{1}{T_{m}^{0}}\right)-\left(\frac{\Delta \tilde{S}_{m}}{\phi_{1}} \frac{1}{\Delta \tilde{H}_{f}}\right)
$$

We can refer the first term in Eq. (12) to be the degree of the depression of melting point $\left(D_{m}\right)$.

$$
D_{m}=\frac{1}{\phi_{1}}\left(\frac{1}{T_{m}}-\frac{1}{T_{m}^{0}}\right)=Q+\left(\frac{\Delta \tilde{S}_{m}}{\phi_{1}} \frac{1}{\Delta \tilde{H}_{f}}\right)
$$

The $D_{m}$ is expected to be reduced in the case where the entropy of mixing has a negative sign. We obtained the empirical equation for $\Delta S_{m i x}^{c} / n_{T}$ in Fig. 3a with the unit $(\mathrm{cal} / \mathrm{mol} \mathrm{K})$ as follows.

$$
\frac{\Delta S_{m i x}^{c}}{n_{T}}=-A \phi_{1} \phi_{2}
$$

where $n_{T}$ is the total number of moles; $n_{T}=n_{1}+n_{2}, n_{1}$ and $n_{2}$ is the number of moles of PEI and PVA, respectively. $A$ is determined to be 2.3 for PVA/PEI blends. A similar value of $A$ is determined for PVA/PEI/GO systems. Then, the molar entropy of mixing is obtained as the following.

$$
\Delta \tilde{S}_{m}=\frac{\partial \Delta S_{m i x}^{c}}{\partial n_{2}}=A \phi_{1}^{2}
$$

Since the entropy of mixing of the blends of PEI and PVA has a negative sign, it can be supposed that the $D_{m}$ would be reduced by the strong hydrogen bonding. However, large depressions of 


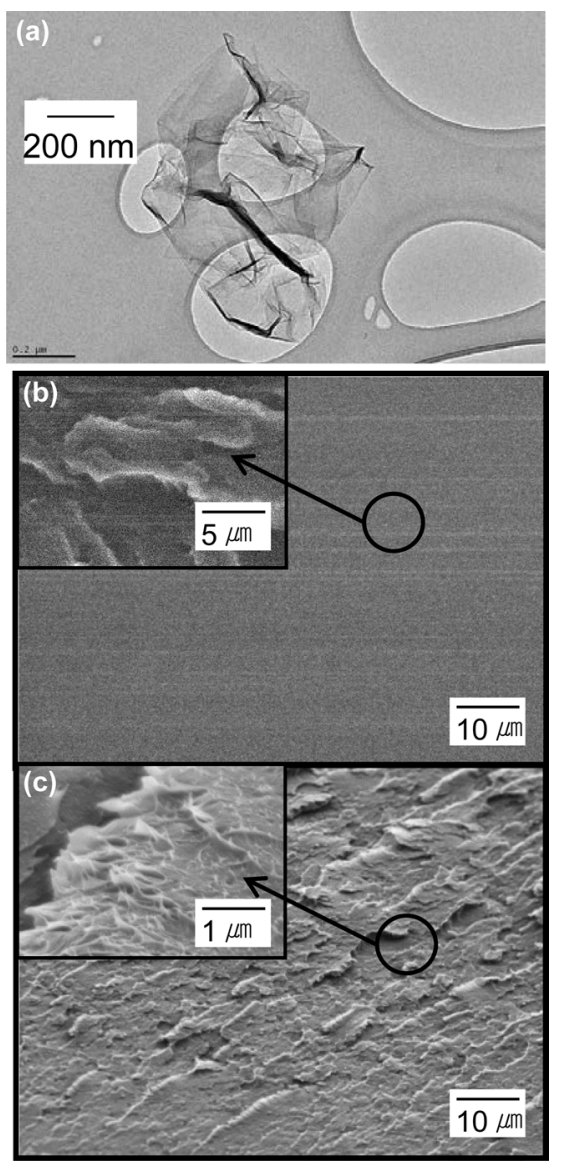

Fig. 7. Morphology of graphene oxide (GO) and the poly(vinyl alcohol) (PVA)/polyethyleneimine (PEI)/GO blends; (a) transmission electron microscopy image of graphene oxide, (b) scanning electron microscopy (SEM) image of the surface of the PVA/PEI (90/10) blends, (c) SEM image of the surface of the PVA/PEI (90/10) blends with $0.7 \mathrm{wt} \% \mathrm{GO}$.

melting points are observed for the blends of PEI and PVA as seen in Fig. 6b. This result points to the fact that the effect of negative entropy may be compensated by the strong enthalpic interaction, as seen in Fig.7. In Fig. 7, the melting temperatures in Fig. $6 \mathrm{~b}$ and the entropy of mixing in Fig. 3a are re-plotted using $Q$ and $\phi_{1} / T_{m}$ as variables. From the slope in Fig. 7, B is determined to be $-37 \mathrm{cal}\left(\mathrm{cm}^{3}\right.$ of PEI) and $\chi$ is -1.34 at $500 \mathrm{~K}$. Eq. (11) reduces to the Nish-Wang equation [49], if the entropy of mixing is neglected. The $\chi$ by Nish-Wang's plot is -0.10 at 500 $K$. So, the magnitude of negative $\chi$ becomes much larger when we consider the entropy term in Eq. (11). Here, it is concluded that the $D_{m}$ in the strong hydrogen bonding system is not reduced by the negative $\Delta S_{m}$, because the magnitude of negative $\chi$ is also increased by the strong hydrogen bonding.

\subsection{Effect of GO on the thermal behaviors of PVA/PEI blends}

GO was prepared from purified conventional flake graphite (19 $\mu \mathrm{m}$ nominal particle size, Asbury Carbon) by the modified Hummers method $[9,17,18]$. The TEM image of the reduced GO sheets suspended on a carbon grid are presented in Fig. 7a,
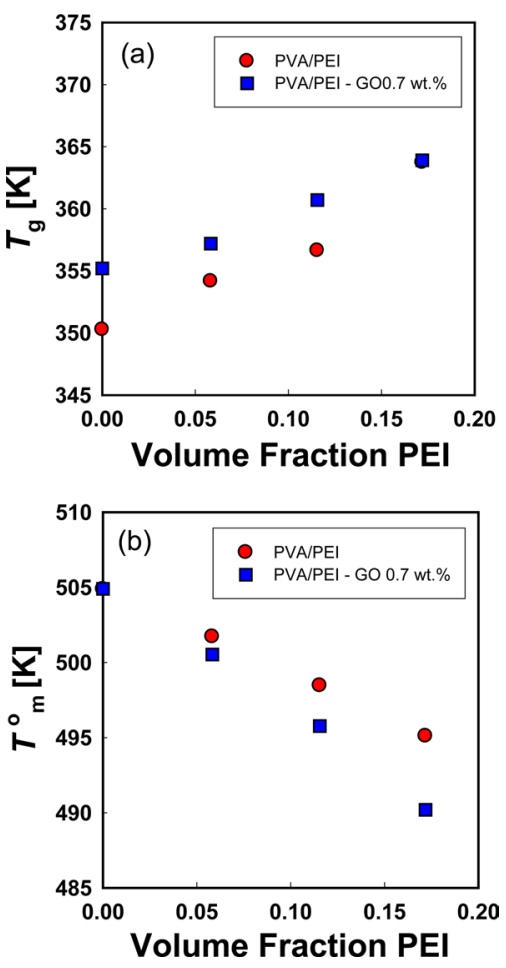

Fig. 8. Effects of graphene oxide on the glass transition temperature and the equilibrium melting temperature of the blends of poly(vinyl alcohol) (PVA) and polyethyleneimine (PEI).

showing the typical image of graphene with electron transparency. We investigated the degree of oxidation of the GO by XPS. The carbon to oxygen atomic ratio of GO was determined to be 1.8 from the XPS spectra. We dispersed $0.7 \mathrm{wt} \% \mathrm{GO}$ into the blends of PVA and PEI. The SEM images of the surface of the blend films are presented in Fig. $7 \mathrm{~b}$ and c. The PVA/PEI blends are thermodynamically miscible to form a single phase, therefore, no appreciable domain is observed in Fig. 7b. A rough layer-like morphology is observed on the surface of the PVA/ $\mathrm{PEI} / \mathrm{GO}$ blends in Fig. 7c. The XRD patterns of the PVA/PEI/ $\mathrm{GO}$ blends show no GO characteristic peak at $2 \theta=9.5^{\circ}$, indicating GO is fully exfoliated in the blends [18].

The $T_{g} s$ and $T_{m}^{0} s$ of PVA/PEI/GO blends are compared to those of PVA/PEI blends in Fig. 8a and b. The $T_{g}$ of PVA is increased about $5 \mathrm{~K}$ by adding GO into PVA. In our previous report [18], the $T_{g}$ of PVA was not changed by adding GO into PVA. We used 86-89 mol\% hydrolyzed PVA in the previous work [18] and 99 mol\% hydrolyzed PVA in this work. Therefore, the hydrogen bonding interaction between PVA and GO may be stronger in this work compared to those in our previous work. This may be evidenced by the increase of the $T_{g} s$ of PVA/PEI blends after adding $0.7 \mathrm{wt} \%$ GO in Fig. 8a. The depressions of $T_{n s}^{0} s$ are more pronounced after adding GO into PVA/PEI blends, as shown in Fig. 9. The results in Figs. 8a and 9 indicate that the hydrogen bonding in PVA/PEI/GO blend systems are pronounced compared to those in the PVA/PEI blend system. We plotted $Q$ versus $\phi_{1} / T_{m}$ for the PVA/PEI/GO blends in Fig. 9. It is reasonable that the mixtures of PEI and GO are treated as diluents for the crystallization of PVA in the PVA/PEI/GO blend systems. So, 


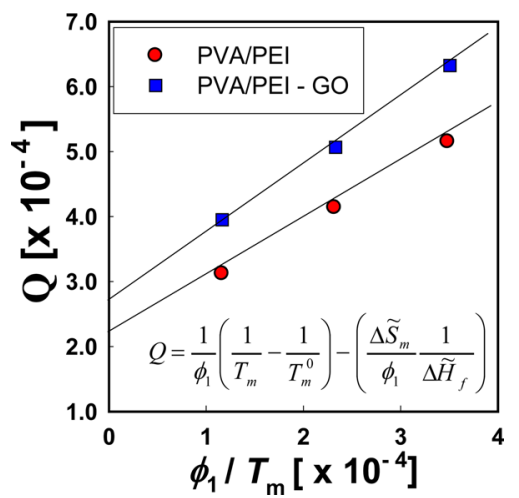

Fig. 9. A plot of quantity $Q$ versus $\phi 1 / T_{m}$ according to Eq. (11). PVA: poly(vinyl alcohol), $\mathrm{PEl}$ : polyethyleneimine.

the $\chi$ in the PVA/PEI/GO blend systems stands for the enthalpic interaction between PVA and the PEI/GO mixture. The $\chi$ in the $\mathrm{PVA} / \mathrm{PEI} / \mathrm{GO}$ ternary blend system was determined to be -1.53 , which is larger than in the PVA/PEI binary blends. The increase of the magnitude of negative $\chi$ by GO may be ascribed to the increase of hydrogen bonding interaction $\left(\chi^{H}\right)$.

\section{Conclusions}

The $T_{g} S$ of PVA/PEI blends were higher than the $T_{g} S$ of either of the component polymers at low concentrations of PEI. These abnormal increases of $T_{g} s$ may be due to the negative entropy of mixing, which is associated with the strong hydrogen bonding between PVA and PEI. The degree of depression of $T_{m s}^{0} S$ was not reduced by the negative entropy of mixing, since strong hydrogen bonding also causes an increase in the magnitude of negative $\chi$ between PVA and PEI. The $T_{g}$ of PVA was inceased significantly by adding $0.7 \mathrm{wt} \% \mathrm{GO}$ into PVA. The magnitude of negative $\chi$ was increased by adding GO into the blends of PVA and PEI.

\section{Acknowledgments}

This work was supported in part by the Graphene Materials/ Components Development Project (10044366) funded by the Ministry of Trade, Industry \& Energy (MI, Korea).

\section{References}

[1] Gao Q, Takizawa J, Kimura M. Hydrophilic non-wovens made of cross-linked fully-hydrolyzed poly(vinyl alcohol) electrospun nanofibers. Polymer, 54, 120 (2013). http://dx.doi.org/10.1016/j. polymer.2012.11.022.

[2] Bessbousse H, Verchère JF, Lebrun L. Characterisation of metalcomplexing membranes prepared by the semi-interpenetrating polymer networks technique. Application to the removal of heavy metal ions from aqueous solutions. Chem Eng J, 187, 16 (2012). http://dx.doi.org/10.1016/j.cej.2011.12.079.

[3] Bunch JS, Verbridge SS, Alden JS, van der Zande AM, Parpia
JM, Craighead HG, McEuen PL. Impermeable atomic membranes from graphene sheets. Nano Lett, 8, 2458 (2008). http://dx.doi. org/10.1021/n1801457b.

[4] Ben Hamouda S, Nguyen QT, Langevin D, Roudesli S. Poly(vinylalcohol)/poly(ethyleneglycol)/poly(ethyleneimine) blend membranes: structure and $\mathrm{CO}_{2}$ facilitated transport. Comptes Rendus Chimie, 13, 372 (2010). http://dx.doi.org/10.1016/j. crci.2009.10.009.

[5] Ben Hamouda S, Roudesli S. Transport properties of PVA/PEI/ PEG composite membranes: sorption and permeation characterizations. Cent Eur J Chem, 6, 634 (2008). http://dx.doi.org/10.2478/ s11532-008-0053-0.

[6] Rao PS, Sridhar S, Wey MY, Krishnaiah A. Pervaporation performance and Transport phenomenon of PVA blend membranes for the separation of THF/water azeotropic mixtures. Polym Bull, 59, 289 (2007). http://dx.doi.org/10.1007/s00289-007-0769-6.

[7] Dong C, Yuan X, He M, Yao K. Preparation of PVA/PEI ultra-fine fibers and their composite membrane with PLA by electrospinning. J Biomater Sci, Polymer Ed, 17, 631 (2006). http://dx.doi. org/10.1163/156856206777346287.

[8] Matsuyama H, Terada A, Nakagawara T, Kitamura Y, Teramoto M. Facilitated transport of $\mathrm{CO}_{2}$ through polyethylenimine/poly(vinyl alcohol) blend membrane. J Membr Sci, 163, 221 (1999). http:// dx.doi.org/10.1016/S0376-7388(99)00183-0.

[9] Kovtyukhova NI, Ollivier PJ, Martin BR, Mallouk TE, Chizhik SA, Buzaneva EV, Gorchinskiy AD. Layer-by-layer assembly of ultrathin composite films from micron-sized graphite oxide sheets and polycations. Chem Mater, 11, 771 (1999). http://dx.doi. $\operatorname{org} / 10.1021 / \mathrm{cm} 981085 \mathrm{u}$.

[10] Dikin DA, Stankovich S, Zimney EJ, Piner RD, Dommett GHB, Evmenenko G, Nguyen ST, Ruoff RS. Preparation and characterization of graphene oxide paper. Nature, 448, 457 (2007). http:// dx.doi.org/10.1038/nature06016.

[11] Geim AK, Novoselov KS. The rise of graphene. Nat Mater, 6, 183 (2007). http://dx.doi.org/10.1038/nmat1849.

[12] Novoselov KS, Geim AK, Morozov SV, Jiang D, Zhang Y, Dubonos SV, Grigorieva IV, Firsov AA. Electric field effect in atomically thin carbon films. Science, 306, 666 (2004). http://dx.doi. org/10.1126/science.1102896.

[13] Shin D, Bae S, Yan C, Kang J, Ryu J, Ahn JH, Hong BH. Synthesis and applications of graphene electrodes. Carbon Lett, 13, 1 (2012). http://dx.doi.org/10.5714/CL.2012.13.1.001.

[14] Kim KS, Zhao Y, Jang H, Lee SY, Kim JM, Kim KS, Ahn JH, Kim P, Choi JY, Hong BH. Large-scale pattern growth of graphene films for stretchable transparent electrodes. Nature, 457, 706 (2009). http://dx.doi.org/10.1038/nature07719.

[15] Kim H, Abdala AA, Macosko CW. Graphene/polymer nanocomposites. Macromolecules, 43, 6515 (2010). http://dx.doi. org/10.1021/ma100572e.

[16] Stankovich S, Dikin DA, Dommett GHB, Kohlhaas KM, Zimney EJ, Stach EA, Piner RD, Nguyen ST, Ruoff RS. Graphenebased composite materials. Nature, 442, 282 (2006). http://dx.doi. org/10.1038/nature04969.

[17] Jun SI, Lee HS. Negative normal stress differences in graphene/ polycarbonate composites. Appl Phys Lett, 100,164108 (2012). http://dx.doi.org/10.1063/1.4704929.

[18] Kim HM, Lee JK, Lee HS. Transparent and high gas barrier films based on poly(vinyl alcohol)/graphene oxide composites. Thin Solid Films, 519, 7766 (2011). http://dx.doi.org/10.1016/j. 
tsf.2011.06.016

[19] Nair RR, Wu HA, Jayaram PN, Grigorieva IV, Geim AK. Unimpeded permeation of water through helium-leak: tight graphene-based membranes. Science, 335, 442 (2012). http://dx.doi.org/10.1126/ science.1211694.

[20] Liang J, Huang Y, Zhang L, Wang Y, Ma Y, Guo T, Chen Y. Molecular-level dispersion of graphene into poly(vinyl alcohol) and effective reinforcement of their nanocomposites. Adv Funct Mater, 19, 2297 (2009). http://dx.doi.org/10.1002/adfm.200801776.

[21] Choperena A, Painter P. Hydrogen bonding in polymers: effect of temperature on the $\mathrm{OH}$ stretching bands of poly(vinylphenol). Macromolecules, 42, 6159 (2009). http://dx.doi.org/10.1021/ma900928z.

[22] Pinal R. Entropy of mixing and the glass transition of amorphous mixtures. Entropy, 10, 207 (2008). http://dx.doi.org/10.3390/entropy-e10030207.

[23] Kuo SW. Hydrogen-bonding in polymer blends. J Polym Res, 15, 459 (2008). http://dx.doi.org/10.1007/s10965-008-9192-4.

[24] Zheng W, Simon SL. Confinement effects on the glass transition of hydrogen bonded liquids. J Chem Phys, 127, 194501 (2007). http:// dx.doi.org/10.1063/1.2793787.

[25] Yi JZ, Goh SH. Miscibility and interactions in poly(n-propyl methacrylate)/poly(vinyl alcohol) blends. Polymer, 46, 9170 (2005). http://dx.doi.org/10.1016/j.polymer.2005.07.011.

[26] He Y, Zhu B, Inoue Y. Hydrogen bonds in polymer blends. Prog Polym Sci, 29, 1021 (2004). http://dx.doi.org/10.1016/j.progpolymsci.2004.07.002.

[27] Feldstein MM, Roos A, Chevallier C, Creton C, Dormidontova EE. Relation of glass transition temperature to the hydrogen bonding degree and energy in poly(N-vinyl pyrrolidone) blends with hydroxyl-containing plasticizers: 3 . Analysis of two glass transition temperatures featured for PVP solutions in liquid poly(ethylene glycol). Polymer, 44, 1819 (2003). http://dx.doi.org/10.1016/ S0032-3861(03)00046-6.

[28] Kuo SW, Xu H, Huang CF, Chang FC. Significant glass-transition-temperature increase through hydrogen-bonded copolymers. J Polym Sci B, 40, 2313 (2002). http://dx.doi.org/10.1002/ polb.10292.

[29] Lagarón JM, Giménez E, Saura JJ, Gavara R. Phase morphology, crystallinity and mechanical properties of binary blends of high barrier ethylene: vinyl alcohol copolymer and amorphous polyamide and a polyamide-containing ionomer. Polymer, 42, 7381 (2001). http://dx.doi.org/10.1016/S0032-3861(01)00204-X.

[30] Park JS, Park JW, Ruckenstein E. A dynamic mechanical and thermal analysis of unplasticized and plasticized poly(vinyl alcohol)/ methylcellulose blends. J Appl Polym Sci, 80, 1825 (2001). http:// dx.doi.org/10.1002/app.1278.

[31] Hassan CM, Peppas NA. Structure and morphology of freeze/ thawed PVA hydrogels. Macromolecules, 33, 2472 (2000). http:// dx.doi.org/10.1021/ma9907587.

[32] Laurence C, Berthelot M. Observations on the strength of hydrogen bonding. Perspect Drug Discovery Des, 18, 39 (2000). http:// dx.doi.org/10.1023/A:1008743229409.

[33] Parada LG, Cesteros LC, Meaurio E, Katime I. Miscibility in blends of poly(vinyl acetate-co-vinyl alcohol) with poly $(\mathrm{N}, \mathrm{N}-$ dimethylacrylamide). Polymer, 39, 1019 (1998). http://dx.doi.
org/10.1016/S0032-3861(97)00389-3.

[34] Schneider HA. Conformational entropy contributions to the glass temperature of blends of miscible polymers. J Res Natl Inst Stand Technol, 102, 229 (1997). http://www.archive.org/details/jresv102n2p229.

[35] Coleman MM, Painter PC. Hydrogen bonded polymer blends. Prog Polym Sci, 20, 1 (1995). http://dx.doi.org/10.1016/0079-6700 (94)00038-4.

[36] Prinos J, Panayiotou C. Glass transition temperature in hydrogenbonded polymer mixtures. Polymer, 36, 1223 (1995). http://dx.doi. org/10.1016/0032-3861(95)93924-B.

[37] Painter PC, Graf JF, Coleman MM. Effect of hydrogen bonding on the enthalpy of mixing and the composition dependence of the glass transition temperature in polymer blends. Macromolecules, 24, 5630 (1991). http://dx.doi.org/10.1021/ma00020a023.

[38] Feng H, Feng Z, Shen L. A high resolution solid-state n.m.r. and d.s.c. study of miscibility and crystallization behaviour of poly(vinyl alcohol)poly(N-vinyl-2-pyrrolidone) blends. Polymer, 34, 2516 (1993). http://dx.doi.org/10.1016/0032-3861(93)90581-T.

[39] Zhang X, Takegoshi K, Hikichi K. High-resolution solid-state ${ }^{13} \mathrm{C}$ nuclear magnetic resonance study on poly(vinyl alcohol)/ poly(vinylpyrrolidone) blends. Polymer, 33, 712 (1992). http:// dx.doi.org/10.1016/0032-3861(92)90326-R.

[40] Fox TG. Influence of diluent and of copolymer composition on the glass temperature of a polymer system. Bull Am Phys Soc, 1, 123 (1956).

[41] Gordon M, Taylor JS. Ideal copolymers and the second-order transitions of synthetic rubbers. I. Non-crystalline copolymers. J Appl Chem, 2, 493 (1952). http://dx.doi.org/10.1002/jctb.5010020901.

[42] Couchman PR, Karasz FE. A Classical thermodynamic discussion of the effect of composition on glass-transition temperatures. Macromolecules, 11, 117 (1978). http://dx.doi.org/10.1021/ ma60061a021.

[43] Kwei TK. The effect of hydrogen bonding on the glass transition temperatures of polymer mixtures. J Polym Sci: Polym Lett Ed, 22, 307 (1984). http://dx.doi.org/10.1002/pol.1984.130220603.

[44] Atkins PW. Physical Chemistry. 3rd ed., W.H. Freeman, New York, NY (1986).

[45] Flory PJ. Principles of Polymer Chemistry. 9th ed., Cornell University Press, London, UK (1975).

[46] Painter PC, Shenoy SL, Bhagwagar DE, Fishburn J, Coleman MM. Effect of hydrogen bonding on the melting point depression in polymer blends where one component crystallizes. Macromolecules, 24, 5623 (1991). http://dx.doi.org/10.1021/ma00020a022.

[47] Gibbs JH, DiMarzio EA. Nature of the glass transition and the glassy state. J Chem Phys, 28, 373 (1958). http://dx.doi.org/ $10.1063 / 1.1744141$.

[48] Hoffman JD, Weeks JJ. Melting process and the equilibrium melting temperature of polychlorotrifluoroethylene. J Res Natl Bur Stand A: Phys Chem, 66A, 13 (1962). http://dx.doi.org/10.6028/ jres.066A.003.

[49] Nishi T, Wang TT. Melting point depression and kinetic effects of cooling on crystallization in poly(vinylidene fluoride)-poly(methyl methacrylate) mixtures. Macromolecules, 8, 909 (1975). http:// dx.doi.org/10.1021/ma60048a040. 